\title{
Comparing soft robotic affective touch to human and brush affective touch
}

\author{
C.Y.Zheng ${ }^{1}$, K-J. Wang ${ }^{2}$, M. Wairagkar ${ }^{3}$, M. Von Mohr ${ }^{4}$, E.Lintunen ${ }^{1}$, K. Fotopoulou ${ }^{5}$
}

\begin{abstract}
Affective touch is important for maintaining emotional bonds and providing comfort. In this pilot study, we developed a silicone pneumatic soft robotic haptic device (S-CAT) to provide affective touch and compared its performance with commonly used brush and human affective touch. The S-CAT device simulates the attributes of CToptimal affective touch in terms of velocity, temperature and force. In 22 participants we administered touch on their forearm from the S-CAT device (robot), a human hand and a soft brush at $6 \mathrm{~cm} / \mathrm{s}$ (CT-optimal speed) and $36 \mathrm{~cm} / \mathrm{s}$ (non-CT optimal speed). We collected subjective ratings on pleasantness and intensity, as well as electroencephalography (EEG) responses. The results showed that pleasantness and intensity ratings depend on velocity of the touch. Moreover, S-CAT touch delivered at these different velocities elicits similar subjective ratings to using a human hand or brush. Findings point to the potential for soft robotic haptic devices to modulate subjective and electrophysiological response in a similar way to more natural, human touch.
\end{abstract}

\section{INTRODUCTION AND THE SOFT ROBOTIC DEVICE}

Human affective touch is known to be psychologically beneficial. Gentle, caress-like touch from soft brush with a velocity of $1-10 \mathrm{~cm} / \mathrm{s}$, an applied force around $0.22-0.5 \mathrm{~N}$, and skin temperature optimally activates the $\mathrm{C}$ Tactile (CT) system[1], and has shown to be strongly correlated with reported pleasantness[2], as well as reduce negative experiences[2]. We developed a soft robotic haptic device, S-CAT, which refers to soft robotic CT-optimal affective touch, using pneumatic silicone material to simulate the attributes of CT- optimal affective touch in terms of velocity, temperature and applied force. The device is comprised of 8 $1 \times 5.5 \mathrm{~cm}$ air cells that are individually controllable by digital valves. Inflating adjacent cells with an overlap time creates a rippling effect resembling stroking of skin. We assessed the performance of this S-CAT device by comparing CT-optimal touch generated by robot, brush and human. Although previous study has examined the electroencephalography (EEG) response toward caress like tactile stimulation from a fabric based robotic device[3], this is the first study to observe the effect of soft robotic affective touch on EEG.

\section{METHODS}

We compared six experimental conditions (3 types of touch stimulations: human, brush, robot at 2 tactile speeds: slow $6 \mathrm{~cm} / \mathrm{s}$ vs fast $36 \mathrm{~cm} / \mathrm{s}$ ) in 22 healthy adult participants. The study was approved by Royal College of Art and participants gave written consent. Tactile stimulations were

Royal College of Art, London W12 7FN UK ( yan.zheng@network.rca.ac.uk). ${ }^{2}$ University of Pittsburgh, 15260, USA (kew88@pitt.edu). ${ }^{3}$ Imperial College London and UK Dementia Research Institute, London SW7 1AL, UK (m.wairagkar@ic.ac.uk). ${ }^{4}$ Royal Holloway University of London, TW20 0EX, UK ( mariana.vonmohr@rhul.ac.uk). ${ }^{5}$ University College London, WC1E 7HB UK (a.fotopoulou@ucl.ac.uk). delivered by left hand fingers strokes of a female experimenter and soft robotic device at approximately $35 \sim 37^{\circ} \mathrm{C}$, and soft brush. Each experimental condition consisted of 8 blocks of 12s stimulation followed by $6 \mathrm{~s}$ no stimulation. Tactile strokes were applied back and forth on the dorsal forearm, with an applied force of $\sim 0.48 \mathrm{~N}$. Participants kept their eyes closed during the stimulations.

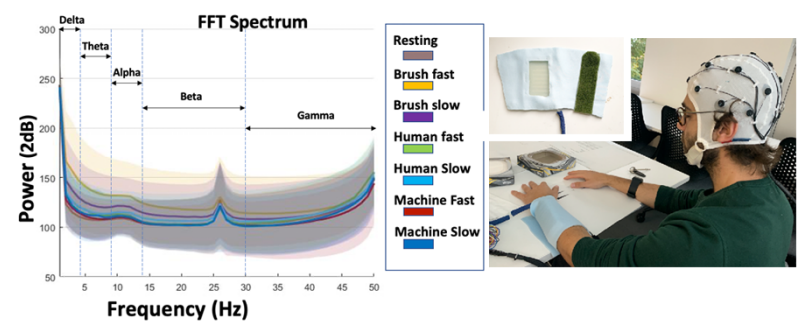

Figure 1. (L) Parietal lobe EEG power spectrum under different touch stimuli.

(R) Wearable soft robotic (S-CAT) device and experimental set-up

Participants rated the level of perceived pleasantness and activation (i.e., intensity / arousal) on an analogue visual guide after each experimental condition. EEG from 16 channels, sampled at $125 \mathrm{~Hz}$ (using OpenBCI) was recorded (Fig. 1, R).

We conducted repeated measures ANOVA specifying stimulation type (human, brush, robot) and speed (slow, fast) separately on pleasantness and activation / arousal ratings. For EEG analysis, we compared band powers of delta $(0.5-4 \mathrm{~Hz})$, theta $(4-8 \mathrm{~Hz})$, alpha $(8-13 \mathrm{~Hz})$, beta $(13-30 \mathrm{~Hz})$ and gamma $(30-50 \mathrm{~Hz})$ in different stimulations and speeds in different brain regions using Friedman test with Bonferroni correction.

\section{RESUlts AND Discussion}

As we expected, subjective ratings showed that participants perceived slow touch to be more pleasant than fast touch irrespective of the stimulation type, and in contrast, fast vs. slow touch was perceived as more arousing. There was no statistically significant difference in perceived pleasantness or arousal between the stimulation type. EEG results showed that band powers of slow robot touch were similar to slow human and slow brush touch (Fig. 1, L). No statistically significant difference was observed in the EEG band powers of these tactile stimulations. This pilot study results suggest that the S-CAT soft robotic device can have a potential to produce pleasantness and EEG response comparable to skin-to-skin and brush affective touch and could be used in applications for psychological benefit.

\section{REFERENCES}

[1] L. S. Löken et al., 'Coding of pleasant touch by unmyelinated afferents in humans', Nat Neurosci, vol. 12, no. 5, pp. 547-548, 2009

[2] M. von Mohr et al., 'The soothing function of touch: affective touch reduces feelings of social exclusion', Scientific Reports, vol. 7, no. 1, p. 13516, 2017

[3] G. Valenza et al., 'EEG oscillations during caress-like affective haptic elicitation', Psychophysiology, vol. 55, no. 10, p. e13199, 2018 